\title{
Overexpression of the transcription factor NF-YC9 confers abscisic acid hypersensitivity in Arabidopsis
}

\author{
Chao Bi ${ }^{1} \cdot$ Yu Ma $^{1} \cdot$ Xiao-Fang Wang $^{1} \cdot$ Da-Peng Zhang ${ }^{1}$
}

Received: 24 May 2017 / Accepted: 13 September 2017 / Published online: 18 September 2017

(C) The Author(s) 2017. This article is an open access publication

\begin{abstract}
Nuclear factor Y (NF-Y) family proteins are involved in many developmental processes and responses to environmental cues in plants, but whether and how they regulate phytohormone abscisic acid (ABA) signaling need further studies. In the present study, we showed that over-expression of the NF-YC9 gene confers ABA hypersensitivity in both the early seedling growth and stomatal response, while down-regulation of $N F-Y C 9$ does not affect $\mathrm{ABA}$ response in these processes. We also showed that overexpression of the NF-YC9 gene confers salt and osmotic hypersensitivity in early seedling growth, which is likely to be directly associated with the ABA hypersensitivity. Further, we observed that NF-YC9 physically interacts with the ABA-responsive bZIP transcription factor ABA-INSENSITIVE5 (ABI5), and facilitates the function of ABI5 to bind and activate the promoter of a target gene EM6. Additionally, NF-YC9 up-regulates expression of the $A B I 5$ gene in response to ABA. These findings show that NF-YC9 may be involved in $\mathrm{ABA}$ signaling as a positive regulator and likely functions redundantly together with other NF-YC members, and support the model that the NF-YC9 mediates ABA signaling via targeting to and aiding the ABA-responsive transcription factors such as ABI5.
\end{abstract}

Chao Bi and Yu Ma have contributed equally to this work.

Electronic supplementary material The online version of this article (doi:10.1007/s11103-017-0661-1) contains supplementary material, which is available to authorized users.

Da-Peng Zhang

zhangdp@tsinghua.edu.cn

1 MOE Systems Biology and Bioinformatics Laboratory, Center for Plant Biology, School of Life Sciences, Tsinghua University, Beijing 100084, China
Keywords Abscisic acid signaling $\cdot$ Arabidopsis thaliana $\cdot$ Nuclear factor NF-YC9 $\cdot$ ABA-INSENSITIVE5 $(\mathrm{ABI} 5) \cdot$ Seedling growth $\cdot$ Stomata

\section{Introduction}

The phytohormone abscisic acid (ABA) regulates plant growth, development, and responses to a variety of abiotic stress (Finkelstein et al. 2002; Adie et al. 2007; Cutler et al. 2010). Numerous regulators of ABA signaling, including receptors for $\mathrm{ABA}$, have been identified, revealing the functional mechanism of this phytohormone from signal perception to downstream gene expression (for review, see Cutler et al. 2010). However, given that ABA signaling is a highly complex signaling pathway, many other components remain to be identified to fully understand the complex ABA signaling network.

Nuclear factor Y (NF-Y), also called CCAAT box combination factor (CBF) or heme activated protein (HAP), is a class of transcription factors that widely exist in yeast, animals and plants. NF-Y consists of three different subunits, the NF-YA (CBF-B or HAP2), NF-YB (CBF-A or HAP3) and NF-YC (CBF-C or HAP5) (Mantovani 1999). In plants, each of the three subunits has multiple members (Stephenson et al. 2007; Siefers et al. 2009; Laloum et al. 2013). In Arabidopsis thaliana, there are ten members of NF-YA subfamily, 13 members of NF-YB subfamily, and 13 members of NF-YC subfamily (Siefers et al. 2009). The NF-YA, NF-YB and NF-YC subunits usually form a huge number of heterotrimer complex, which functions to bind DNA and regulate gene expression (Gusmaroli et al. 2001; Petroni et al. 2012). It has been believed that, while the NF-Y proteins have retained high degrees of similarity, especially in the residues necessary for NF-Y complex formation and DNA 
binding, they may be evolving unique, even antagonistic, regulatory roles for some processes likely through formation of different complexes with different NF-Y members (Stephenson et al. 2007; Siefers et al. 2009; Laloum et al. 2013). While the NF-Y family proteins function as a hetero-trimer complex, a recent report showed that Arabidopsis NF-YC1/ HAP5A directly bound the promoter of a target gene regulated the gene expression in freezing response (Shi et al. 2014), revealing that a single NF-Y subunit may function alone without formation of a hetero-trimer complex. Additionally, increasing evidence showed that the NF-Y members may work together or alone to interact with and help other transcription factors to function in gene expression regulation (Wright et al. 1995; Benatti et al. 2008; Yamamoto et al. 2009; Liu and Howell 2010; Kumimoto et al. 2013; Yotsui et al. 2013; Yeap et al. 2017). Recent reports showed that NF-Y members are involved in chromatin remodeling (Cao et al. 2014; Hou et al. 2014; Tang et al. 2017). These findings further support the functional diversity and complexity of the NF-Y family proteins.

Recent studies showed importance of the NF-Y family proteins in ABA signaling (Warpeha et al. 2007; Yamamoto et al. 2009; Kumimoto et al. 2013; Shi et al. 2014; Siriwardana et al. 2014; Liu et al. 2016) and ABA-mediated stress responses (Nelson et al. 2007; Li et al. 2008, 2013; Leyva-González et al. 2012; Sato et al. 2014; Shi et al. 2014). NF-YA5 mutants were shown to be hypersensitive to ABA during seed germination (Warpeha et al. 2007) and susceptible to drought, but overexpression of NF-YA5 increased drought tolerance in an ABA-dependent manner (Li et al. 2008). In a complete NF-Y family analysis, all the ten Arabidopsis NF-YA members were systematically over-expressed, and the results showed that some transgenic lines were hypersensitive and others were hyposensitive to $\mathrm{ABA}$ in seed germination, in which the overexpression lines of NF-YA2, NF-YA4, NF-YA5, NFYA7, NF-YA8, and NF-YA10 were ABA hypersensitive, and those of NF-Y1 and NFYA9 were ABA hyposensitive (Leyva-González et al. 2012; Mu et al. 2013). The opposing ABA-related phenotypes suggest that this closely-related homologues evolved distinct roles during ABA-mediated seed germination (Siriwardana et al. 2014). The NF-YC members were also recently reported to be involved in ABA signaling. Different NF-YC proteins were shown to have unique and opposing functions in ABA-mediated seed germination, in which mutants of NF-YC4 were hypersensitive, but mutants of NF-YC3 were hyposensitive, to ABA, and mutants of NF-YC9 displayed wild-type ABA-related phenotype, during seed germination (Kumimoto et al. 2013). However, a most recent report showed that the seeds of an NF-YC9overexpression line have lower testa and endosperm rupture rate, showing that overexpression of NF-YC9 results in ABA hypersensitivity in seed germination (Liu et al. 2016).
Therefore, whether and how NF-Y family proteins regulate ABA signaling needs further studies to understand the complex mechanism of ABA function.

In the present study, we showed that overexpression of NF-YC9 enhances the sensitivity to ABA and salt and osmotic stresses during early seedling growth, and also promotes stomatal response to $\mathrm{ABA}$, though the knockdown mutants of NF-YC9 show wild-type ABA-related phenotypes, suggesting that NF-YC9 may positively regulate ABA signaling but likely with a functional redundancy. We further showed that NF-YC9 interacts with and improves activities of an ABA responsive bZIP transcription factor ABI5 and enhances expression of $A B I 5$ gene in response to ABA. These findings help to understanding functional role of the NF-YC proteins in ABA signaling.

\section{Materials and methods}

\section{Plant materials and growth conditions}

Arabidopsis thaliana ecotype Col-0 was used as the wild type materials. The mutants $n f-y c 9-1$ (SALK_058903) and $n f-y c 9$-2 (SALK_069632) were obtained from the Arabidopsis Biological Resource Center (ABRC, Columbus, OH, USA). The homozygous mutants were identified by PCR using gene-specific primers (see Supplemental Table S1 in Supplemental Data). The mutant $n f-y c 9-1$ and the NF-YC9 overexpression transgenic lines OE1 and OE6, generous gifts from Dr. Hao Yu (Department of Biological Sciences, National University of Singapore), were previously identified and described (Hou et al. 2014). We verified the NF-YC9 mRNA levels in the $n f-y c 9$ mutants and $N F-Y C 9$ overexpression lines OE1 and OE6 by quantitative RT-PCR analysis with the gene-specific primers ( see Supplemental Table S2 in Supplemental Data).

Plants were grown in a growth chamber at $22{ }^{\circ} \mathrm{C}$ on MS medium (Phyto Technology Laboratories, Shawnee Mission, KS, USA, product No. M519) in a growth chamber at $\sim 80 \mu \mathrm{mol}$ photons $\mathrm{m}^{-2} \mathrm{~s}^{-1}$ or in compost soil at $\sim 120 \mu \mathrm{mol}$ photons $\mathrm{m}^{-2} \mathrm{~s}^{-1}$ lighted by cool-white fluorescent lamps under a $16 \mathrm{~h}$-light $/ 8 \mathrm{~h}$-dark photoperiod and $60 \%$ relative humidity.

\section{Phenotypic analysis}

Phenotypic analysis was performed as described previously (Wu et al. 2009; Shang et al. 2010). For the post-germination growth and cotyledon greening assays, seeds (about 100) were sterilized and plated on MS medium that contained 3\% sucrose and $0.8 \%$ agar ( $\mathrm{pH}$ 5.9) supplemented with different concentrations of ABA or $\mathrm{NaCl}$ or D-mannitol (Amresco, Solon, OH, USA). The seeds were chilled at $4{ }^{\circ} \mathrm{C}$ for 3 days 
before being placed at $22^{\circ} \mathrm{C}$ under light conditions (16-h light/8-h dark). The fresh weight of single seedling, the root length and the cotyledon greening rate were measured at the indicated times. Leaves of the 4-week-old seedlings grown in the soil were used for investigation of the stomatal closure. To observe ABA-induced stomatal closure, detached leaves were immersed in a buffer containing $50 \mathrm{mM} \mathrm{KCl}$ and $10 \mathrm{mM}$ MES-KOH (pH 6.15) under a halogen cold light source (Chongqing Optec Instrument Co., Lt, Chongqing, China) for $3 \mathrm{~h}$. Subsequently, the detached leaves were transferred into the fresh buffer solution $(50 \mathrm{mM} \mathrm{KCl}$ and $10 \mathrm{mM}$ MES-KOH pH 6.15) with $20 \mu \mathrm{M}( \pm)$ ABA (Sigma, Saint Louis, MO, USA) for $2 \mathrm{~h}$ before the stomatal apertures were measured. To assay ABA-inhibited stomatal opening, the plants were kept in the dark for $24 \mathrm{~h}$ before the detached leaves were immersed in the same buffer described as above supplemented with $20 \mu \mathrm{M}( \pm)$ ABA for $2 \mathrm{~h}$ under the cold light, and the apertures were measured. In addition, epidermal strips were removed from the abaxial surface of the detached leaves and photographed with the Olympus DIC microscope (Olympus, BX51, Japan).

\section{Yeast two-hybrid assay}

The interaction between NF-YC9 and ABI5 proteins was assayed by a yeast two-hybrid system, of which the experimental procedures were described by the manufacturer (Clontech, Mountain View, CA, USA). The full-length coding sequence of $N F-Y C 9$ was cloned to pGADT7 vector fused with the DNA activation domain (AD), and the fulllength coding sequence of $A B I 5$ was cloned to pGBKT7 vector fused to GAL4 binding domain (BD). The primers used for the related constructs are listed in Supplemental Table S3. The different combination pairs of the constructs were used to co-transform yeast strain AH109. Co-transformants were plated on Leu-Trp-deficient medium (SD-2) and Leu-Trp-His-Ade-deficient medium (SD-4) and grown for 5-7 days at $30^{\circ} \mathrm{C}$. The yeast cells co-transformed with the construct pairs AD plus BD-ABI5 and BD plus ADNF-YC9 were used as negative controls, and the yeast cells co-transformed with the construct pairs BD-p53 plus AD-T were taken as a positive control.

\section{Electrophoretic mobility shift assay (EMSA)}

EMSA was performed essentially as previously described (Liu et al. 2012, 2013; Shang et al. 2010) and by using the Light Shift Chemiluminescent EMSA kit (Thermo Scientific, Waltham, MA, USA, product No. 89,880) according to the manufacturer instructions. The NF-YC9-6His and ABI5-6His recombinant proteins were produced in E. coli strain BL21. The full-length coding sequence of the $N F$ $Y C 9$ or $A B I 5$ gene was cloned into the Sal I/EcoR I sites of
pMAL-c5X vector (NEB), respectively. The 5' end biotinlabeled probes used for this EMSA were amplified by PCR using the primer pairs described in Supplemental Table S4, and unlabeled fragments of the same sequences were used as the competitors. The labeled probes ( $20 \mathrm{fmol})$ were incubated with the ABI5-6His recombinant proteins $(1 \mu \mathrm{g})$ in a binding buffer solution ( $25 \mathrm{mM}$ HEPES, $40 \mathrm{mM} \mathrm{KCl}, 5 \mathrm{mM}$ $\mathrm{MgCl}_{2}, 1 \mathrm{mM}$ DTT, $1 \mathrm{mM}$ EDTA, and $8 \%$ glycerol, $\mathrm{pH}$ 8.0) in the presence of $1 \mu \mathrm{g} \mu \mathrm{L}^{-1}$ of poly(deoxyinosinicdeoxycytidylic) sodium salt [poly(dI-dC)] (Sigma, USA, product No. P4929) for $30 \mathrm{~min}$ at room temperature. The NF-YC9-6His protein was applied at a gradient of concentrations $(1-4 \mu \mathrm{g})$. The DNA-protein reaction mixtures were separated on a 5\% non-denaturing polyacrylamide gel (acry lamide:bisacrylamide $=19: 1$ ). The detailed procedures were described in the manufacturer instructions of the EMSA kit.

\section{Luciferase complementation imaging (LCI)}

LCI assay was performed to further verify the interaction between NF-YC9 and ABI5 in Nicotiana benthamiana leaves according to previously described procedures (Chen et al. 2008; Shang et al. 2010). The full-length coding sequence of $N F-Y C 9$ and $A B I 5$ were constructed to the C-terminus of Luc (CLuc) vector and the N-terminus of Luc (NLuc) vector, respectively. Primers used for the vector construction were listed in Supplemental Table S5. The constructs were transformed into Agrobacterium tumefaciens GV3101. Bacteria were suspended in the infiltration buffer solution $(0.2 \mathrm{mM}$ acetosyringone, $10 \mathrm{mM} \mathrm{MgCl}$, and $10 \mathrm{mM} \mathrm{MES}$ ) to identical concentrations $(\mathrm{OD} 600=0.6)$. Bacterial suspensions were infiltrated into young but fully expanded leaves of the seven-week old $N$. benthamiana leaves using a needleless syringe. It is noteworthy that the amounts of the constructs were the same between the different treatments and controls for each group of assay. After infiltration, plants were grown firstly under dark for $24 \mathrm{~h}$ and then with $16 \mathrm{~h} \mathrm{light/day} \mathrm{for}$ $60 \mathrm{~h}$ at room temperature. The luciferase (LUC) activity was observed with a CCD imaging apparatus (Andor iXon, Andor Technology, Belfast, UK). The leaves were sprayed with $0.5 \mathrm{mM}$ luciferin and were placed in darkness for $3 \mathrm{~min}$ before luminescence detection.

\section{Assay of the transcription factor-promoter interaction in $N$. benthamiana leaves}

This assay was performed essentially as previously described (Shang et al. 2010). The NF-YC9 and ABI5 proteins were used for the effectors. The promoter of the EM6 gene was amplified by PCR using the primer pairs listed in Supplemental Table S6. The PCR product was cloned to generate the reporter construct, promoter of EM6 linked to luciferase (ProEM6::LUC). The full-length coding sequence 


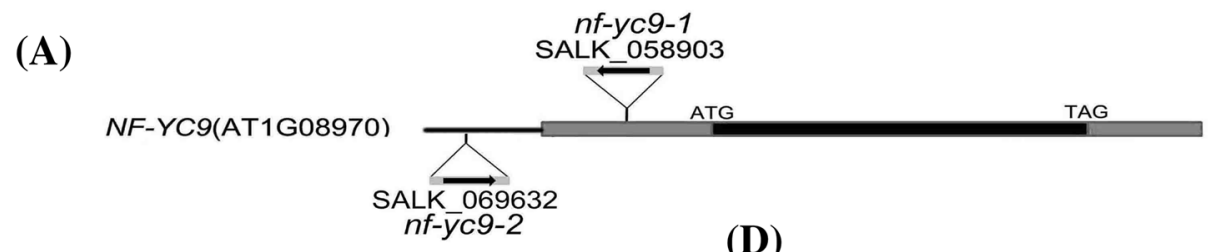

(B)

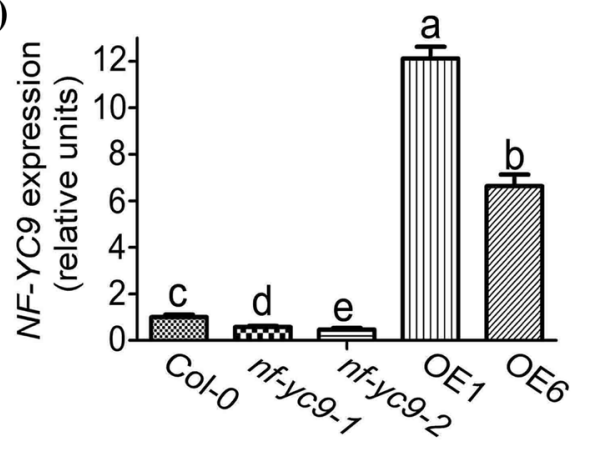

(C)

ABA
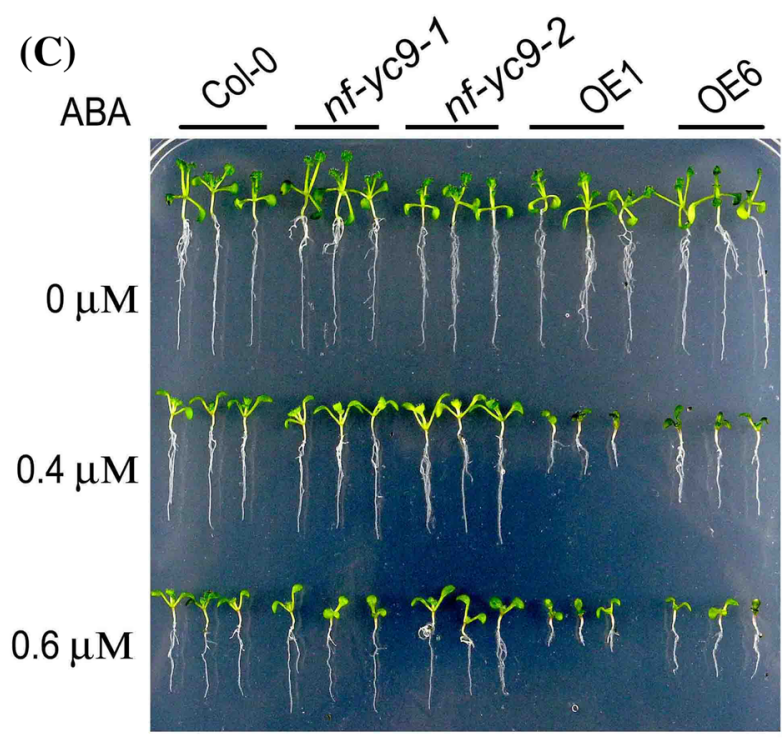

(E)

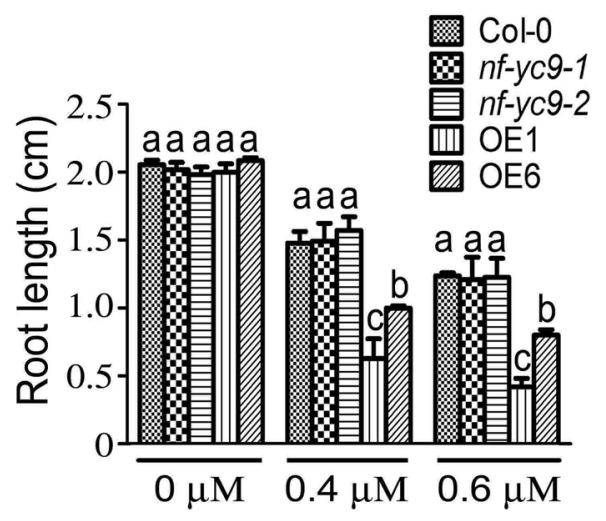

of the NF-YC9 and ABI5 gene were fused to the pMDC 85 and pCAMBIA1300-221 vector as the effector constructs (35S::NF-YC9, 35S::ABI5), which were used to transform, respectively, A. tumefaciens strain GV3101. The transformant-bacteria suspensions were infiltrated into the 7-week
ABA
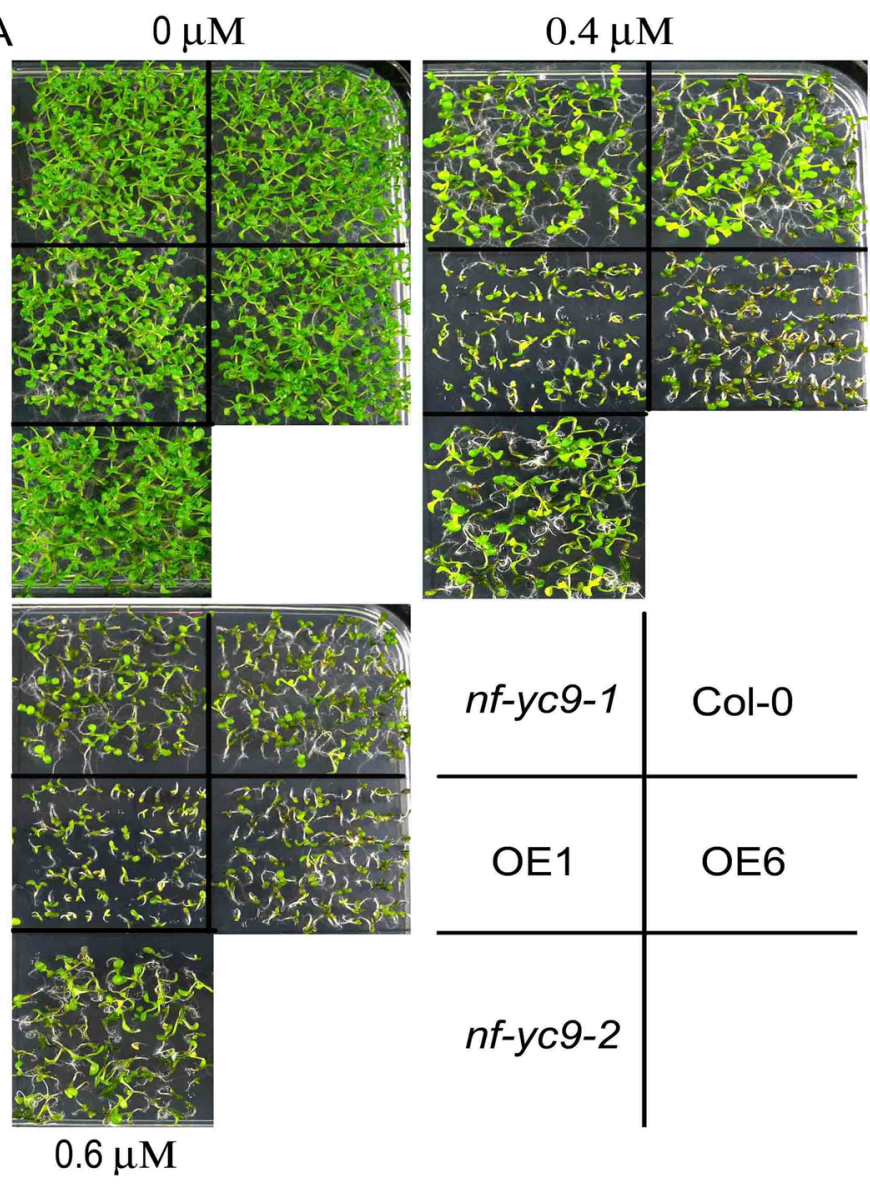

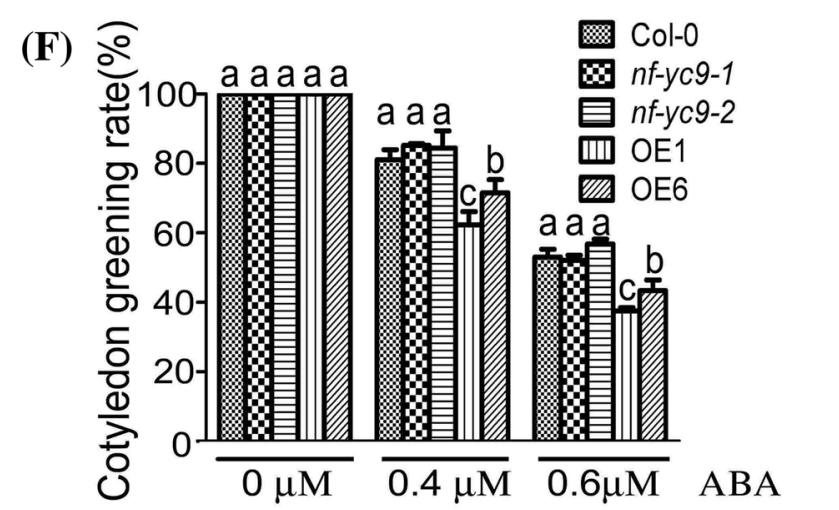

old $N$. benthamiana plants. It is noteworthy that the amounts of the A. tumefaciens strain containing different constructs were the same between the different treatments and controls for each group of assay. After infiltration, plants were grown firstly under dark for $24 \mathrm{~h}$ and then with $16 \mathrm{~h}$ light/day for 
\Fig. 1 Over-expression of $N F-Y C 9$ results in ABA hypersensitivity but down-regulation of $N F-Y C 9$ expression shows no effect on ABA response during early seedling growth. a Structures of the NF-YC 9 gene are shown with the T-DNA insertion site in $n f-y c 9$ 1 (SALK_058903) and $n f-y c 9-2$ (SALK_069632). Black box: exon; grey box: $5^{\prime}$ and $3^{\prime}$ UTR; thick line: the promoter region from the start code ATG of the NF-YC9 gene. The black arrowhead indicates the orientation of the T-DNA insertion. b Quantitative RT-PCR analysis of NF-YC9 gene expression in wild-type Col-0, $n f-y c 9-1, n f-y c 9$ 2 mutants and the $N F-Y C 9$ overexpression transgenic lines $\mathrm{OE} 1$ and OE6. Expression level of NF-YC9 in all the genetic materials was normalized to that of Actin2/8, and the expression level of $N F-Y C 9$ in the wild-type Col- 0 was set to 1 . Each value is the mean $\pm \mathrm{SE}$ of three independent biological experiments, and different letters indicate significant differences at $\mathrm{P}<0.05$ (Duncan's multiple range test). c Root growth of the different genotypes (the wild-type Col-0, $n f-y c 9$ land $n f-y c 9-2$ knockdown mutants, and the NF-YC9 overexpression transgenic lines OE1 and OE6) growing on $( \pm)$ ABA-free $(0 \mu \mathrm{M})$ and ABA-containing $(0.4$ and $0.6 \mu \mathrm{M})$ MS medium. Seeds were directly sown on the medium, and the root growth was investigated 14 days after a stratification at $4{ }^{\circ} \mathrm{C}$ for 3 days. The experiments were repeated four times with similar results. d Post-germination growth of the different genotypes as shown in (c). Seeds were directly sown on $( \pm)$ ABA-free $(0 \mu \mathrm{M})$ or ABA-containing $(0.4$ and $0.6 \mu \mathrm{M})$ MS medium, and the growth was investigated 10 days after a stratification at $4{ }^{\circ} \mathrm{C}$ for 3 days. The experiments were repeated four times with similar results. e Statistical analysis of root length of the different genotypes described in (c). Each value is the mean \pm SE of three independent biological experiments, and different letters indicate significant differences at $\mathrm{P}<0.05$ (Duncan's multiple range test) when comparing values within the same ABA concentration. f Statistical analysis of cotyledon greening rate of the different genotypes as described in (d). Green cotyledons were recorded 7 days after a stratification at $4{ }^{\circ} \mathrm{C}$ for 3 days. Each value is the mean $\pm \mathrm{SE}$ of three independent biological experiments, and different letters indicate significant differences at $\mathrm{P}<0.05$ (Duncan's multiple range test) when comparing values within the same ABA concentration

$60 \mathrm{~h}$ at room temperature. The LUC activity was observed with a CCD imaging apparatus (Andor iXon, Andor Technology, Belfast, UK). The leaves were sprayed with $0.5 \mathrm{mM}$ luciferin and were placed in darkness for 3 min before luminescence detection.

\section{Bimolecular imaging of fluorescent complementation (BiFC) assay}

BiFC assays were performed essentially as previously described (Shang et al. 2010). We used the wild type Col-0 plants to prepare Arabidopsis protoplast. The full-length coding sequences of $\mathrm{NF}-\mathrm{YC} 9$ and $A B I 5$ were constructed to the pUC-SPYNE and pUC-SPYCE vector (cYFP-NF-YC9 or ABI5-nYFP), respectively. The primers used for constructing the related plasmids are listed in Supplemental Table S7. The YFP fluorescence was imaged under a Leica confocal laser scanning microscope (Zeiss LSM780, Germany).
Transient expression in Arabidopsis protoplasts to assay subcellular localization of NF-YC9 protein

Transient expression in the Arabidopsis protoplasts was performed essentially as previously described (Shang et al. 2010). NF-YC9 was fused with the green fluorescent protein (GFP) protein (NF-YC9-GFP), and the known nuclearand cytoplasmic protein PYR1 (Park et al. 2009) was fused with the red fluorescent protein (RFP) as a control (PYR1RFP). The corresponding cDNAs were amplified by PCR (see Supplemental Table S8 for the primers). Protoplasts were isolated from the leaves of 3- to 4-week old plants of Arabidopsis (ecotype Col-0). Fluorescence of GFP or RFP was observed by confocal microscope (Zeiss LSM780, Germany).

\section{Quantitative RT-PCR}

For quantitative RT-PCR (qRT-PCR) analysis, total RNA was isolated from the 3-weeks-old seedlings. Seeds were sown in MS medium, chilled for 3 days at $4{ }^{\circ} \mathrm{C}$ and placed at $22^{\circ} \mathrm{C}$ under a 16 -h light/8-h dark photoperiod for 3 weeks. Using Total RNA Rapid Extraction Kit (BioTeke, Beijing, China, product No. RP3301), the RNA samples for qRTPCR were prepared, treated with RNase-free DNaseI (NEB) at $37^{\circ} \mathrm{C}$ for $30-60 \mathrm{~min}$ to degrade genomic DNA, and purified by using RNA Purification Kit (BioTeke, Beijing, China, product No. RP1801). Total RNA $(2 \mu \mathrm{g})$ were subjected to first-strand cDNA synthesis using a kit according to the manufacturer's instructions (Roche, Mannheim, Germany, product No.AS095014379012001). The primers used for qRT-PCR are listed in Supplemental Table S9. Analysis was performed using the Bio-Rad Real-Time System CFX96TM C1000 Thermal Cycler (Bio-Rad, USA). Amplification of the Actin 2/8 genes was used as an internal control. The qRTPCR conditions were as follows: $10 \mathrm{~min}$ of denaturation at $95^{\circ} \mathrm{C}$, followed by 45 cycles of $10 \mathrm{~s}$ of denaturation at $95^{\circ} \mathrm{C}$, $5 \mathrm{~s}$ of annealing at $55^{\circ} \mathrm{C}$ and a $10 \mathrm{~s}$ extension at $72^{\circ} \mathrm{C}$. The cDNA was amplified using SYBR Premix ExTaq (TaKaRa, Dalian, China). All experiments were repeated at least three times along with three biologically independent repetitions.

\section{Results}

\section{Over-expression of $\mathrm{NF}-\mathrm{YC} 9$ results in $\mathrm{ABA}$ hypersensitivity in early seedling growth and stomatal response}

The T-DNA insertion homozygous mutants of the NF-YC9 gene, $n f-y c 9-1$ and $n f-y c 9-2$, were shown to be knockdown alleles (Fig. 1a, b). The NF-YC9 gene was significantly 
over-expressed in the transgenic lines OE1 and OE6 (Fig. 1b). We observed that, in the absence of exogenously applied ABA, there was no significant difference found between the wild-type and $N F$-YC9-overexpressing lines (OE1 and OE6) plants in the early seedling growth (as indicated by both primary root length and cotyledon greening rate), while the early seedling growth of the OE1 and OE6 lines was significantly more reduced than that of the wildtype seedlings in the ABA-containing medium (Fig. 1c-f).

The promotion of stomatal closure and inhibition of stomatal opening have been believed to be two distinct ABA-mediated processes, which can minimize water transpiration from the leaves under drought conditions (Zhu 2002; Kwak et al. 2008). We observed that, in the presence of exogenously applied ABA, the OE1 and OE6 plants exhibited significantly ABA-hypersensitive phenotypes in both ABA-induced promotion of stomatal closure and inhibition of stomatal opening compared with wild-type plants (Fig. 2a-d).

These data suggest that NF-Y9 may be positively involved in ABA signaling in both early seedling growth and stomatal response. However, we found that the knockdown mutants $n f-y c 9-1$ and $n f-y c 9-2$ displayed wild-type phenotypes in early seedling growth and stomatal response (Figs. 1, 2), suggesting that the Arabidopsis NF-YC family proteins may function redundantly in these ABA-mediated processes.

\section{Over-expression of $\mathrm{NF}$-YC9 leads to salt and osmotic hypersensitivity during early seedling growth}

We observed that, in the absence of exogenously applied $\mathrm{NaCl}$ or D-mannitol (used as a osmotic reagent), there was no significant difference found between the wild-type and $N F-Y C 9$-overexpressing lines (OE1 and OE6) plants
Fig. 2 Over-expression of $N F$ $Y C 9$ promotes, but down-regulation of $N F-Y C 9$ expression shows no effect on, stomatal response to $\mathrm{ABA}$. a $\mathrm{ABA}$ inhibition of stomatal opening, and b ABA promotion of stomatal closure, in the wild-type Col-0, $n f-y c 9-1$ knockdown mutant and the NF-YC9 overexpression transgenic lines OE1 and OE6. Mature rosette leaves from 3-week-old seedlings were used for the assays. Each value is the mean \pm SE of three independent biological experiments, and different letters indicate significant differences at $\mathrm{P}<0.05$ (Duncan's multiple range test) when comparing values within the same ABA concentration. c Photographs showing the stomatal aperture of the ABAinduced inhibition of stomatal opening in the different genotypes as described in (a). Bars represent $10 \mu \mathrm{m}$. d Photographs showing the stomatal aperture of the ABA-induced promotion of stomatal closure in the different genotypes as described in (b). Bars represent $10 \mu \mathrm{m}$
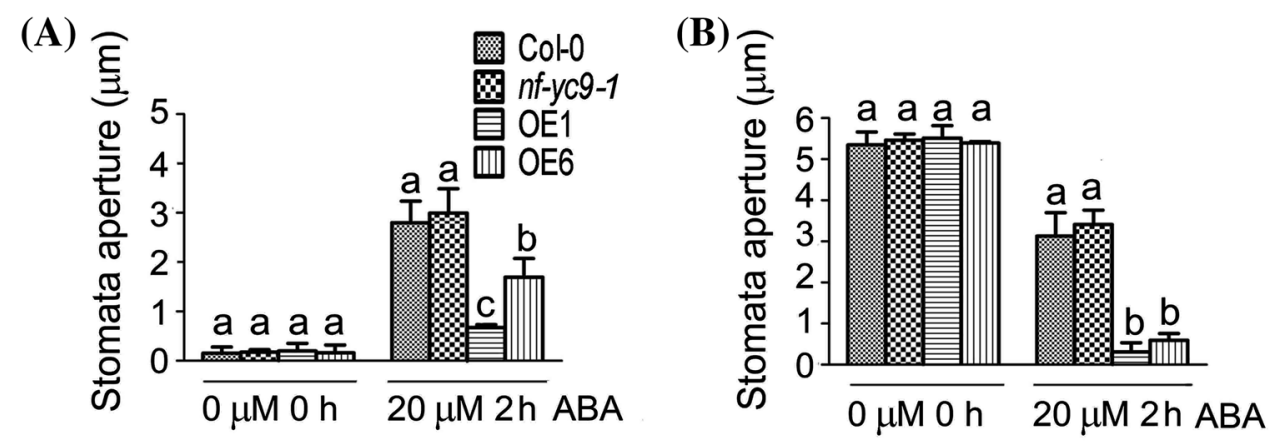

(C)

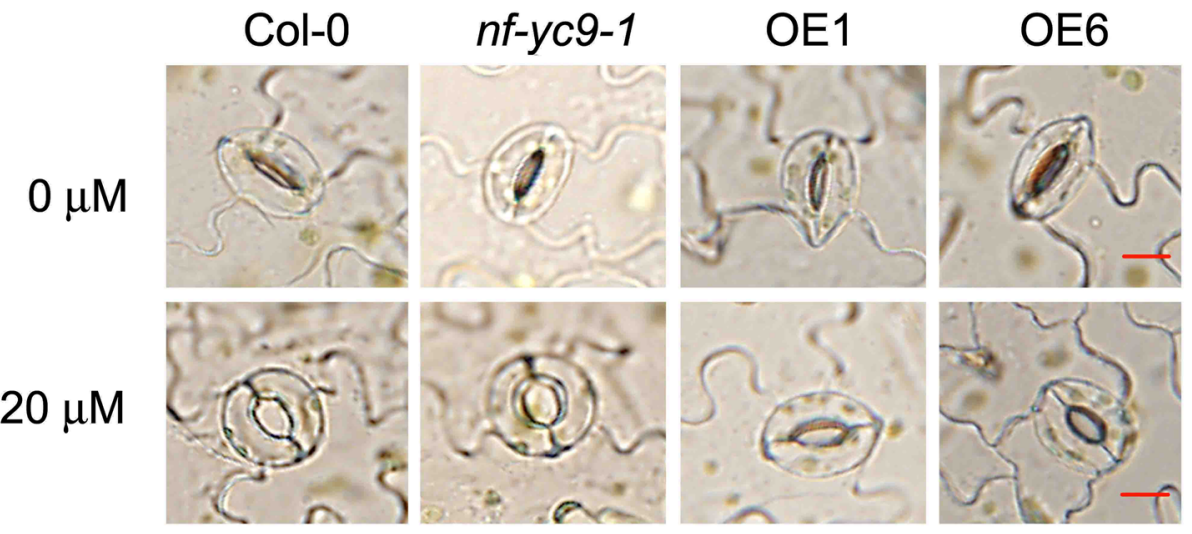

(D)

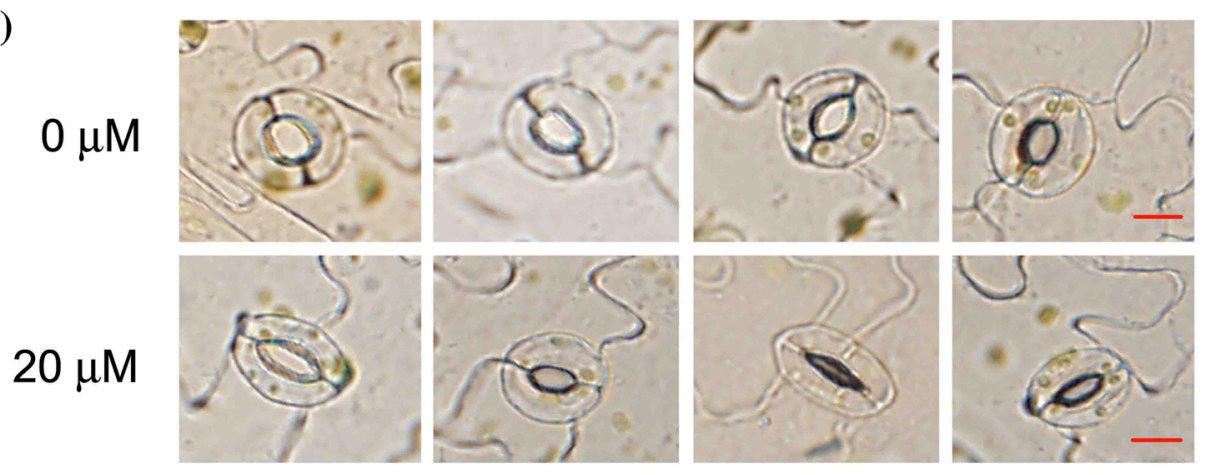


(A) $\mathrm{NaCl}$

$0 \mathrm{mM}$
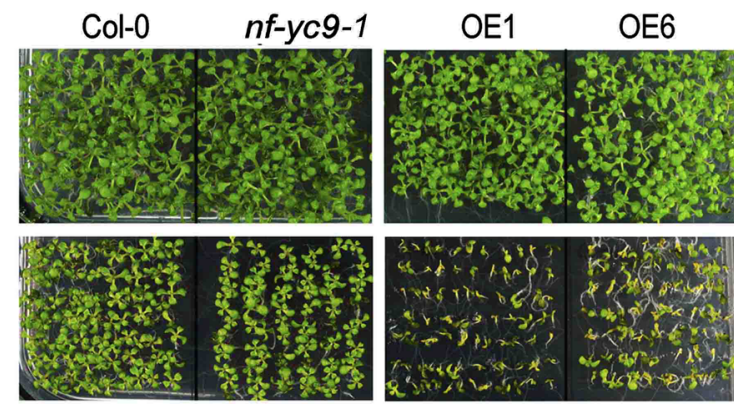

$100 \mathrm{mM}$

$120 \mathrm{mM}$

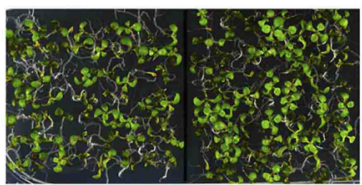

(C)
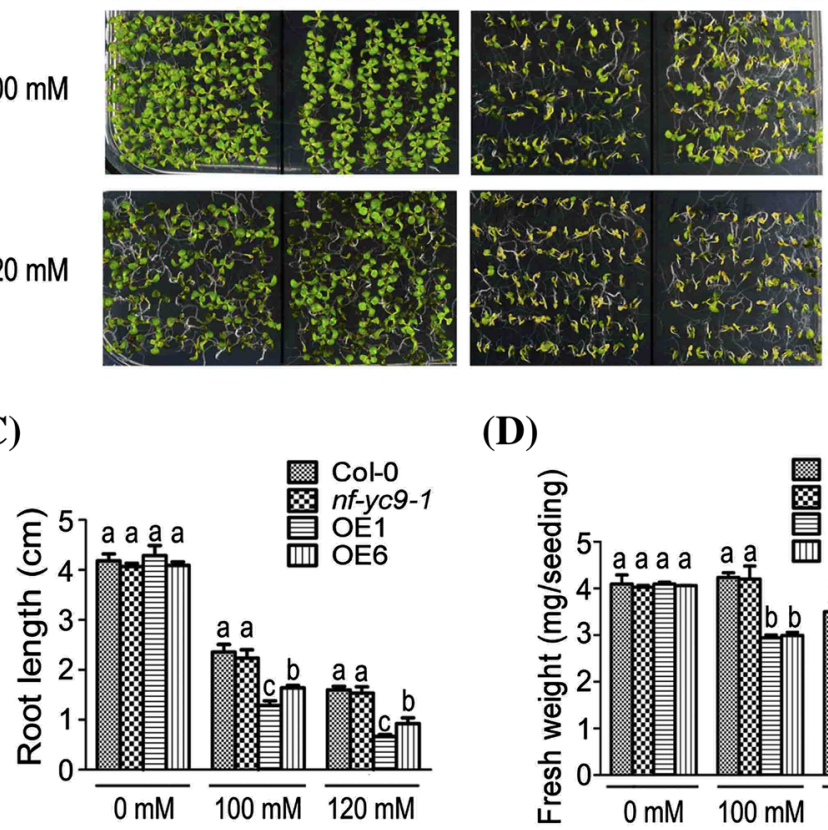

(B) $\mathrm{NaCl}$

$0 \mathrm{mM}$

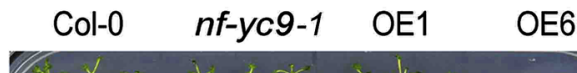

$100 \mathrm{mM}$

$120 \mathrm{mM}$

(D)

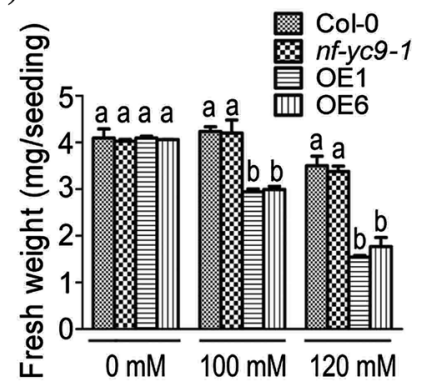

(E)

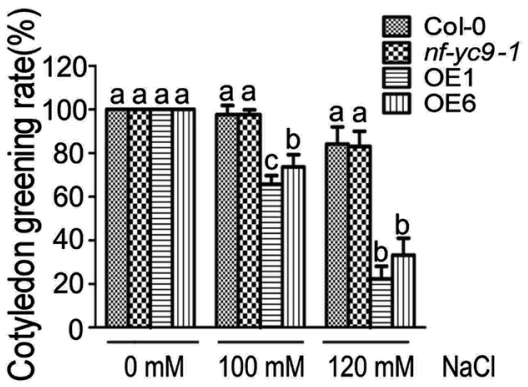

Fig. 3 Over-expression of $N F-Y C 9$ results in salt stress hypersensitivity but down-regulation of $N F-Y C 9$ expression shows no effect on salt response during post-germination growth. a Post-germination growth of the wild-type Col-0, $n f-y c 9-1$ knockdown mutant and the NF-YC9 overexpression transgenic lines OE1 and OE6 plants. Seeds were directly sown on $\mathrm{NaCl}$-free $(0 \mathrm{mM})$ or NaCl-containing $(100 \mathrm{mM}$, and $120 \mathrm{mM}$ ) MS medium, and the growth was investigated 10 days after a stratification at $4{ }^{\circ} \mathrm{C}$ for 3 days. The experiments were repeated four times with similar results. b Root growth of the different genotypes as described in (a) growing on $\mathrm{NaCl}$-free $(0 \mathrm{mM})$ or $\mathrm{NaCl}$-containing $(100$, and $120 \mathrm{mM}) \mathrm{MS}$ medium. Seeds were directly sown on the medium, and the root growth was investigated 14 days after a stratification at $4{ }^{\circ} \mathrm{C}$ for 3 days. The experiments were repeated four times with similar results. c Statistical analysis of root length of the different genotypes as described in (b). Each value is the mean $\pm \mathrm{SE}$

in the early seedling growth (as indicated by primary root growth, plant fresh weight and cotyledon greening), whereas the early seedling growth of the OE1 and OE6 lines was significantly more reduced than that of the wild-type seedlings in the salt- or D-mannitol-containing medium (Figs. 3a-e, 4a-e). Given that both $n f-y c 9$ mutants showed the same phenotypes, the $n f-y c 9-1$ mutant was used as a representative in the following experiments, which displayed wild-type phenotypes in response to these stresses (Figs. 3a-e, 4a-e). These data are consistent with those described above for ABA responses (Figs. 1, 2), suggesting that NF-YC9 may be positively involved in both ABA- and salt-induced osmotic response of three independent biological experiments, and different letters indicate significant differences at $\mathrm{P}<0.05$ (Duncan's multiple range test) when comparing values within the same $\mathrm{NaCl}$ concentration. d Statistical analysis of fresh weight ( $\mathrm{mg} / \mathrm{seedling}$ ) of the different genotypes as described in (a). Each value is the mean \pm SE of three independent biological experiments, and different letters indicate significant differences at $\mathrm{P}<0.05$ (Duncan's multiple range test) when comparing values within the same $\mathrm{NaCl}$ concentration. e Statistical analysis of cotyledon greening rate of the different genotypes as described in (a). Green cotyledons were recorded 7 days after a stratification at $4{ }^{\circ} \mathrm{C}$ for 3 days. Each value is the mean $\pm \mathrm{SE}$ of three independent biological experiments, and different letters indicate significant differences at $\mathrm{P}<0.05$ (Duncan's multiple range test) when comparing values within the same $\mathrm{NaCl}$ concentration

during early seedling growth, and also supporting the idea that the NF-YC family proteins may function redundantly in the ABA- and salt-induced osmotic responses.

\section{Subcellular localization of NF-YC9 protein and expression profile of $\mathrm{NF}-\mathrm{YC} 9$ gene}

The transient expression assay in Arabidopsis protoplasts showed that NF-YC9 fused with GFP localizes to both nucleus and cytoplasm, merged well with a known nuclearand cytoplasmic protein PYR1, which was fused with RFP and is a member of ABA receptors (Park et al. 2009) (Fig. 5a). 
(A)

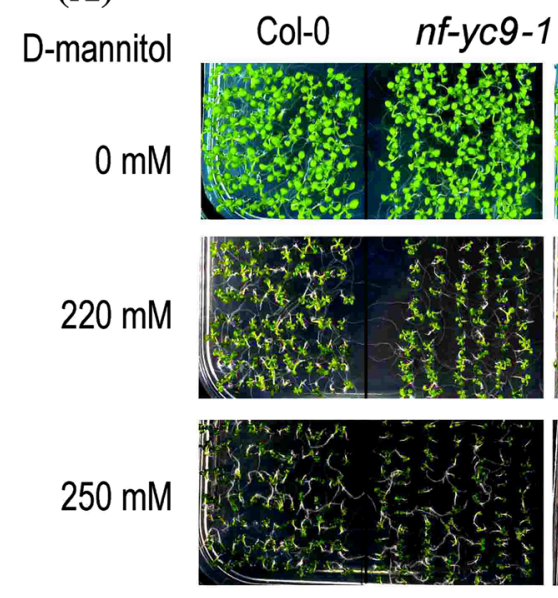

(C)

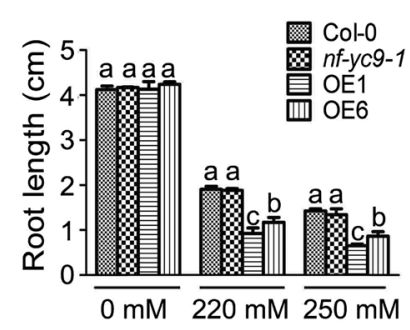

(D)

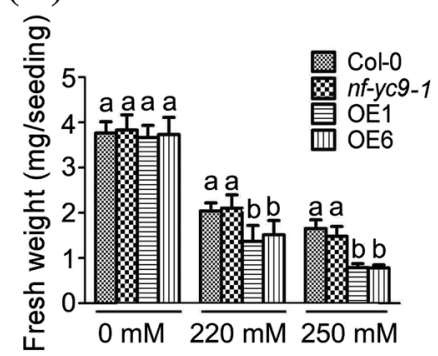

(B)
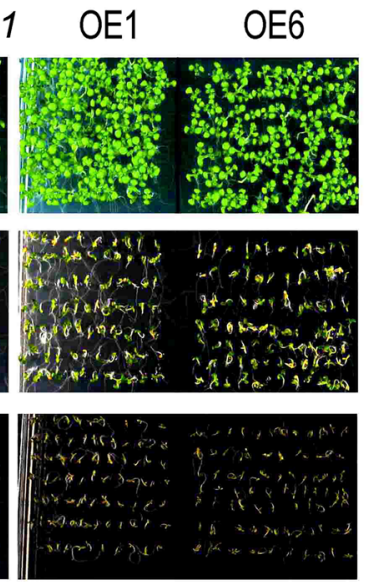

\section{(E)}

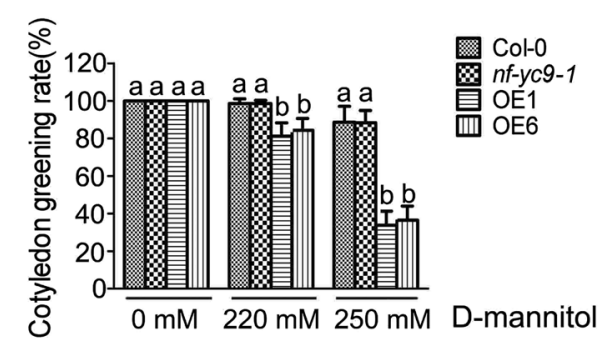

Fig. 4 Over-expression of $N F-Y C 9$ results in osmotic stress hypersensitivity but down-regulation of $N F-Y C 9$ expression shows no effect on osmotic response during post-germination growth. a Post-germination growth of the wild-type Col-0, $n f-y c 9-1$ knockdown mutant and the $N F-Y C 9$ overexpression transgenic lines OE1 and OE6 plants. Seeds were directly sown on D-mannitol-free $(0 \mathrm{mM})$ or D-mannitol-containing $(220$, and $250 \mathrm{mM}) \mathrm{MS}$ medium, and the growth was investigated 10 days after a stratification at $4{ }^{\circ} \mathrm{C}$ for 3 days. The experiments were repeated four times with similar results. b Root growth of the different genotypes as described in (a) growing on D-mannitol-free $(0 \mathrm{mM})$ or D-mannitol-containing (220, and $250 \mathrm{mM}$ ) MS medium. Seeds were directly sown on the medium, and the root growth was investigated 14 days after a stratification at $4{ }^{\circ} \mathrm{C}$ for 3 days. The experiments were repeated four times with similar results. c Statistical analysis of root length of the different genotypes as described in (b). Each value is the mean $\pm \mathrm{SE}$ of three independent biological experiments, and different letters indicate significant differences at $\mathrm{P}<0.05$ (Duncan's multiple range test) when comparing values within the same D-mannitol concentration. d Statistical analysis of fresh weight $(\mathrm{mg} / \mathrm{seedling})$ of the different genotypes as described in (a). Each value is the mean \pm SE of three independent biological experiments, and different letters indicate significant differences at $\mathrm{P}<0.05$ (Duncan's multiple range test) when comparing values within the same D-mannitol concentration. e Statistical analysis of cotyledon greening rate of the different genotypes as described in (a). Green cotyledons were recorded 7 days after a stratification at $4{ }^{\circ} \mathrm{C}$ for 3 days. Each value is the mean $\pm \mathrm{SE}$ of three independent biological experiments, and different letters indicate significant differences at $\mathrm{P}<0.05$ (Duncan's multiple range test) when comparing values within the same D-mannitol concentration
RT-PCR assays showed that the NF-YC9 was ubiquitously expressed in all the organs/tissues with the highest level in dry seeds (Fig. 5b), which is essentially consistent with the expression data found at the public bioinformatics websites AtGenExpress (http://jsp. weigelworld.org/expviz/expviz.jsp) and Arabidopsis eFP Browe (http://bar.utoronto.ca/efp/cgi-bin/efpWeb. cgi?dataSource=Developmental_Map) (see Supplemental Fig. S1 in Supplemental Data). Further, we observed that $N F-Y C 9$ expression level was induced first and then repressed by ABA treatment (Fig. 5c), which may be associated with its role in ABA signaling.

\section{NF-YC9 interacts with ABI5 physically}

To test how NF-YC9 is involved in ABA signaling and based on bioinformatics analysis, we investigated whether NF-YC9 interacts functionally with an ABA-responsive transcription factor ABI5. For testing physical interaction between these two proteins in the yeast two-hybrid system, the full coding sequence of $N F-Y C 9$ was cloned to pGADT7 vector fused with the $\mathrm{AD}$, and the full coding sequence of $A B I 5$ was cloned to pGBKT7 vector fused with the BD. The yeast cells co-transformed with the construct pairs AD-NF-YC9 plus $\mathrm{BD}-\mathrm{ABI} 5$ or BD-p53 plus AD-T (a positive control) 


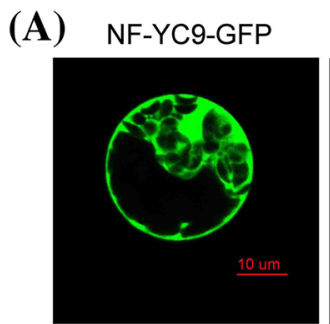

(B)

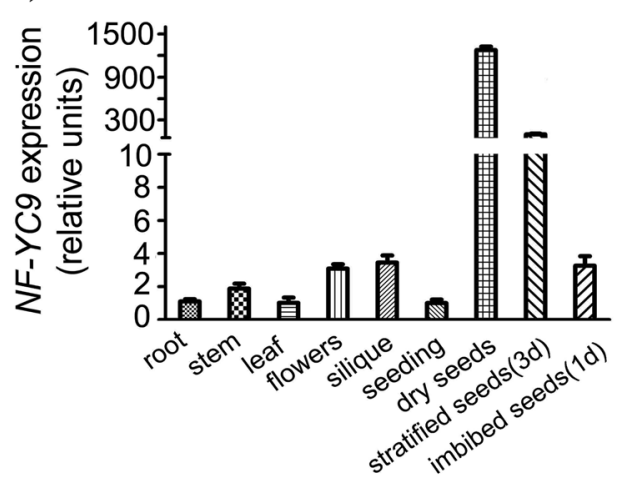

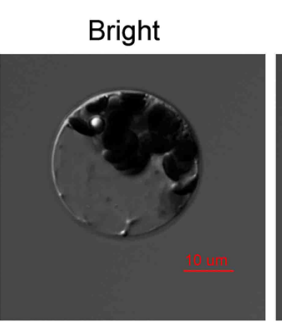

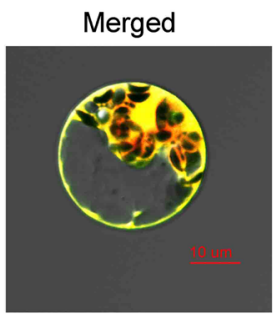

(C)

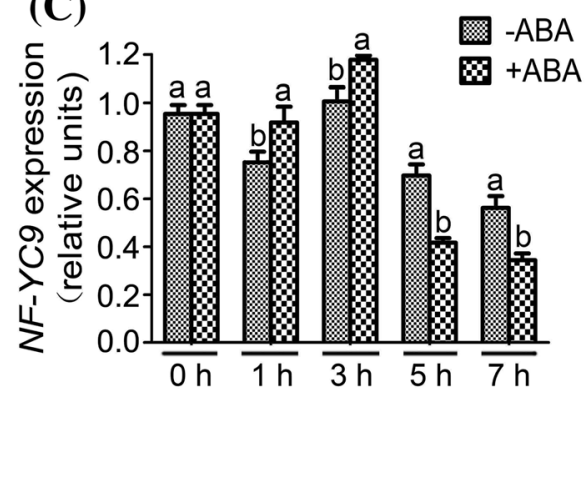

Fig. 5 Subcellular localization of NF-YC9 protein and expression profile of NF-YC9 gene. a NF-YC9 is a nuclear and cytoplasmic dual-localization protein. NF-YC9 was fused with the green fluorescent protein (GFP) protein (NF-YC9-GFP); PYR1 was fused with the red fluorescent protein (RFP) as a control (PYR1-RFP). Subcellular localization was assayed by using the transient expression system in Arabidopsis protoplasts. Bright, bright field; Merged: merged image of the NF-YC9-GFP and PYR1-RFP in the bright field. Bars represent $10 \mu \mathrm{m}$. b Expression profile of $N F-Y C 9$ in various tissues/organs of wild type plants examined by real-time PCR. Relative units for the NF-YC9 expression are normalized relative to the value of root, which is taken as 1 . Each value is the mean $\pm \mathrm{SE}$ of three independent biological experiments. $\mathbf{c}$ The expression levels of $N F-Y C 9$ change in response to ABA treatment. Seedlings of the wild-type Col-0 plants were treated with $100 \mu \mathrm{M}( \pm)$ ABA solution or mock solution $[0 \mu \mathrm{M}$ ( \pm ) ABA solution, as a control], and sampled for gene expression analysis from 0 to $7 \mathrm{~h}$ with the intervals of $2 \mathrm{~h}$ after the ABA treatment. Relative units for the gene expression are normalized relative to the value of wild-type plants at $0 \mathrm{~h}$ with $0 \mu \mathrm{M}( \pm) \mathrm{ABA}$ treatment, which is taken as 1 . The transcript levels of $N F-Y C 9$ were examined using quantitative RT-PCR analysis. Each value is the mean $\pm \mathrm{SE}$ of three independent biological experiments, and different letters indicate significant differences at $\mathrm{P}<0.05$ (Student's $t$ test) when comparing values within the same time point were able to grow in the SD4-dropout medium (lacking Leu, Trp, His, and Ade), while the yeast cells co-transformed with the construct pairs AD plus BD-ABI5 and BD plus AD-NFYC9 (as negative controls), were not able to grow in the SD4-dropout medium, indicating that NF-YC9 interacts with $\mathrm{ABI} 5$ and that the interaction detected in this yeast system is specific and reliable (Fig. 6a).

Next, we used the tobacco LCI system to confirm the interaction between NF-YC9 and ABI5. NF-YC9 and ABI5 were constructed to the $\mathrm{C}$-terminus of Luc (CLuc) and the $\mathrm{N}$-terminus of Luc (NLuc), respectively. NF-YC9-CLuc and ABI5-NLuc co-expressed tobacco leaves displayed significant Luc activity (strong fluorescence), but no Luc activity was detected in the leaves co-infiltrated with three negative control pairs (NLuc plus CLuc, NF-YC9-CLuc plus NLuc or CLuc plus ABI5-NLuc) (Fig. 6b).

We further tested this interaction in the Arabidopsis protoplast system with BiFC. NF-YC9 and ABI5 were constructed to the C-terminus of yellow fluorescent protein (cYFP) and the N-terminus of YFP (nYFP), respectively.
The signal of yellow fluorescent (YFP) were detected by co-expression of cYFP-NF-YC9 with ABI5-nYFP (Fig. 6c). These data from both LCI and BiFC systems consistently verify the interaction between NF-YC9 and ABI5.

\section{NF-YC9 both facilitates ABI5 activity and enhances expression of $A B I 5$ gene in response to $\mathrm{ABA}$}

To test functional significance of the NF-YC9-ABI5 interaction, we used a well-established transient expression assay in N. Benthamiana (Chen et al. 2011; Zhai et al. 2013), in which a direct downstream target gene of ABI5, EM6 (Carles et al. 2002), was used as a candidate target. We constructed a vector of LUC fused with the EM6 promoter from the start codon ATG to the upstream 1973 bp (ProEM6::LUC reporter). The full coding sequence of the $N F-Y C 9$ and $A B I 5$ genes were fused to the pMDC85 and pCAMBIA1300-221 expression vectors as the effector constructs (35S::NF-YC9, 35S::ABI5), respectively. Co-expression of ProEM6::LUC with the $35 \mathrm{~S}:: A B I 5$ showed a significant increase of the 


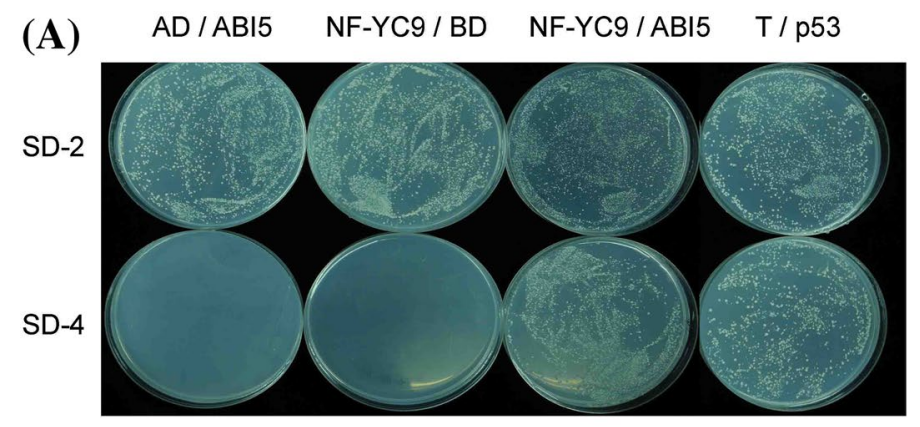

(B)
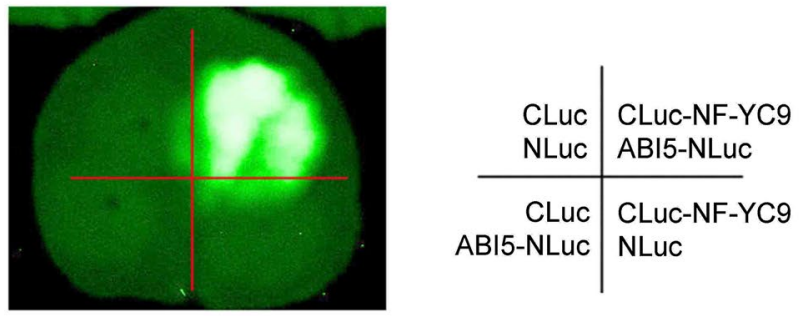

(C)
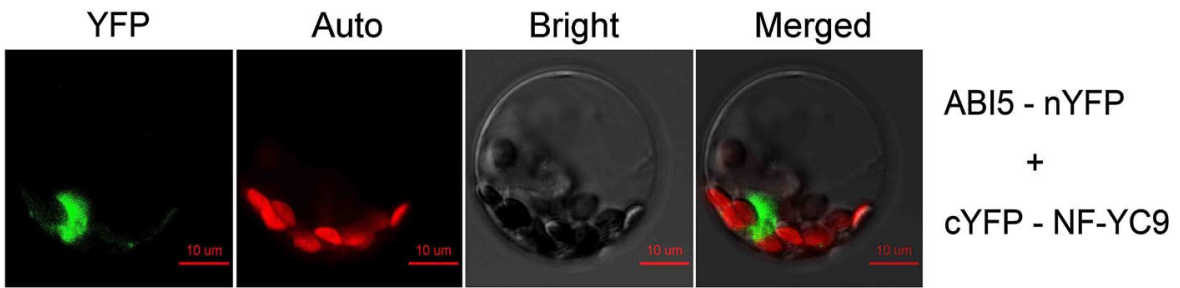

Fig. 6 NF-YC9 interacts with ABI5. a Assays of yeast two-hybrid growth in SD4-drop-out medium (lacking Leu, Trp, His, and Ade) to test the interaction between NF-YC9 and ABI5 protein. The fulllength coding sequence of $N F-Y C 9$ was cloned to pGADT7 vector fused with the $\mathrm{AD}$, and the full-length coding sequence of $A B I 5$ was cloned to pGBKT7 vector fused with the BD. The yeast cells cotransformed with the construct pairs AD-NF-YC9 plus BD-ABI5 or BD-53 plus AD-T (a positive control) were able to grow in the SD4dropout medium (lacking Leu, Trp, His, and Ade), while the yeast cells co-transformed the construct pairs $\mathrm{AD}$ plus BD-ABI5 and $\mathrm{BD}$ plus AD-NF-YC9 (as negative controls) were not able to grow in the SD4-drop-out medium. The experiments were repeated three times with similar results. b Test of the interaction between NF-YC9 and ABI5 by LCI assay. The full-length coding sequence of NF-YC 9 and $A B I 5$ were constructed to the C-terminus of Luc (CLuc) and the

luminescence intensity, indicating that 35S::ABI5 promoted the expression of ProEM6::LUC in this system as expected (Fig. 7a, b). Co-expression combination of 35S::NF-YC9, 35S::ABI5 and ProEM6::LUC enhanced significantly the luminescence intensity of the reporter compared with the co-expression of ProEM6::LUC with the 35S::ABI5 alone (Fig. 7a, b). This observation suggests that NF-YC9 facilitates the function of $\mathrm{ABI} 5$ that activates transcription of the EM6 gene.

We further test this cooperative effect of NF-YC9 and ABI5 on the promoter activity of the target gene EM6 in the
$\mathrm{N}$-terminus of Luc (NLuc) vector, respectively. Tobacco leaves were co-transformed with the construct pairs as described in the right panel. The left panel shows the luciferin fluorescence of the treated leaf. The experiments were repeated three times with similar results. c BiFC assay to test the interactions between NF-YC9 and ABI5 in the Arabidopsis protoplast system. The full-length coding sequence of NF-YC9 and ABI5 were constructed to the C-terminus of YFP (cYFP) and the N-terminus of YFP (nYFP) vector, respectively, and the construct pair was used to co-transform the protoplasts. The fluorescent signal was investigated by a confocal laser scanning microscope. YFP, ABI5-nYFP plus cYFP-NF-YC9 signal; Auto, chlorophyll auto-fluorescent signal; Bright, bright field; Merged, merged image of the YFP signal with chlorophyll auto-fluorescent signal in the bright field. The experiments were repeated three times with similar results

EMSA system using purified ABI5-6His and NF-YC9-6His recombinant proteins (with 6His tag protein as a negative control). Previous work (Carles et al. 2002) showed that ABI5 binds the $E M 6$ promoter sequence called the pEm6.2 probe (AATAAAGTCAGACACGTGGCATGTCACCAA) with the highest affinity. So we chose and synthesized the ATG upstream ( -220 to $-277 \mathrm{bp}$ ) fragment of EM6 promoter region, which includes the pEm6.2 probe sequence and used as the probe in our experiment (by the gene synthesis company GBT, Beijing, China). We found that the sole ABI5-6His protein bound the probe, but the NF-YC9-6His 
protein did not bind the probe (Fig. 7c). The addition of a gradient of amounts of NF-YC9-6His fusion protein promoted the ability of ABI5-6His to bind the DNA probe in a dose-dependent manner (Fig. 7c), indicating that NF-YC9 improves the DNA binding ability of ABI5, consistent with the results from the above transient expression assay in $N$. Benthamiana.

Additionally and interestingly, we found that ABAinduced up-regulation of $A B I 5$ expression was significantly repressed in $n f-y c 9-1$ mutant, but enhanced in NF-YC9overexpressing lines (OE1 and OE6) (Fig. 7d), suggesting that NF-YC9 both facilitates ABI5 activity and enhances expression of $A B I 5$ gene in response to $\mathrm{ABA}$.

\section{NF-YC9 expression level is positively correlated with expression levels of $\mathrm{ABI} 5$ target genes EMIand EM6}

Given that NF-YC9 is ubiquitously expressed in all the organs/tissues with the highest level in dry seeds (Fig. 5b; see Supplemental Fig. S1 in Supplemental Data), it is likely that down-expression of NF-YC9 and over-expression of NF-YC9 would affect transcript levels of genes downstream of ABI5. Early studies indicated that many late embryogenesis abundant (LEA) genes such as Eml and Em6 were $A B I 5$-target genes, as their transcripts were significantly reduced in abi5 mutants (Finkelstein and Lynch 2000; Carles et al. 2002). As expected, transcripts of EMI and EM6 were significantly reduced in $n f-y c 9-1$ mutant, but enhanced in NF-YC9-overexpressing line OE6 (Fig. 7e). Besides, we found that ABA-induced up-regulation of $R D 29 B$ expression (Shinozaki and Yamaguchi-Shinozaki 2007) was significantly repressed in $n f-y c 9-1$ mutant, but enhanced in NF-YC9-overexpressing line OE6 (Fig. 7e), indicating that other ABA-inducible genes are also likely regulated by NF-YC9. Consistent with the above mentioned data, these observations support the idea that NF-YC9 positively regulates $\mathrm{ABI} 5$ transcription activity.

\section{Discussion}

\section{NF-YC9 may be involved in ABA signaling potentially as a positive regulator}

As described above, nuclear factor Y (NF-Y) family proteins NF-YA, NF-YB and NF-YC subunits may function to directly regulate gene expression together as different hetero-trimers or alone as a single NF-Y subunit without formation of a hetero-trimer (Gusmaroli et al. 2001; Stephenson et al. 2007; Siefers et al. 2009; Petroni et al. 2012; Laloum et al. 2013; Shi et al. 2014). They may also function to interact with and help other transcription factors by working together or alone (Wright et al. 1995; Benatti et al. 2008; Yamamoto et al. 2009; Liu and Howell 2010; Kumimoto et al. 2013; Yotsui et al. 2013; Yeap et al. 2017). Some NF-Y members are also involved in chromatin remodeling to modulate gene expression (Cao et al. 2014; Hou et al. 2014; Tang et al. 2017). This complicated mode of action likely allows the functional diversity and complexity of the NF-Y family proteins. Indeed, many members of NF-YA, NF-YB and NF-YC subfamilies have been shown to be involved, positively or negatively, in ABA and stress signaling (Nelson et al. 2007; Warpeha et al. 2007; Li et al. 2008, 2013; Yamamoto et al. 2009; Liu and Howell 2010; Leyva-González et al. 2012; Kumimoto et al. 2013; Sato et al. 2014; Shi et al. 2014; Siriwardana et al. 2014). Among the NF-YC members, whether NF-YC9 is involved in ABA and stress signaling is unclear, because a previous report showed no related phenotypes with a single mutant of $N F-Y C 9$ gene, $n f$ $y c 9$, in seed germination (Kumimoto et al. 2013). However, an overexpression line of $\mathrm{NF}-\mathrm{YC} 9$ has been reported to be ABA hypersensitive in seed germination, as evidenced by lower testa and endosperm rupture rate (Liu et al. 2016). Especially, the seed germination of the $n f-y c 3 n f-y c 9$ double mutant and $n f-y c 3 n f y c 4 n f-y c 9$ triple mutant were insensitive to ABA, whereas, surprisingly, the germination of the single mutant $n f-y c 4$ and double mutants $n f-y c 3 n f y c 4$ and $n f y c 4 n f-y c 9$ was hypersensitive to ABA (Kumimoto et al. 2013). Therefore it is confusing whether and how these NF-YC members regulate ABA signaling.

In the present experiment, we confirmed that down-regulation of NF-YC9 has no effect on ABA response in seed germination (data not shown), which was consistent with the previous report (Kumimoto et al. 2013). Importantly, we observed that, while down-regulation of NF-YC9 affects neither early seedling growth nor stomatal movement in response to ABA, over-expression of the NF-YC9 gene confers ABA hypersensitivity in both early seedling growth and stomatal response (Figs. 1, 2). We also showed that overexpression of the NF-YC9 gene confers salt and osmotic hypersensitivity in early seedling growth (Figs. 3, 4), which is likely to be directly associated with the ABA hypersensitivity. In fact, it is well known that ABA accumulates in salt stress as in other abiotic stresses, and increased levels of ABA result in inhibition of seed germination and are required for tolerance of seedling growth to salt (Zhu 2002, 2003; Shinozaki et al. 2003). Therefore, the salt and osmotic hypersensitivity resulting from $N F-Y C 9$ over-expression in post-germination growth should be attributed to ABA hypersensitivity in the situation of the salt- and osmotic-induced high levels of ABA. This point of view may be supported by the observations that the abil abi2 double knockout mutant, hypersensitive to ABA, is also hypersensitive to salt stress in seed germination and post-germination growth (Jiang et al. 2015). The same phenomenon was observed in 
(A)

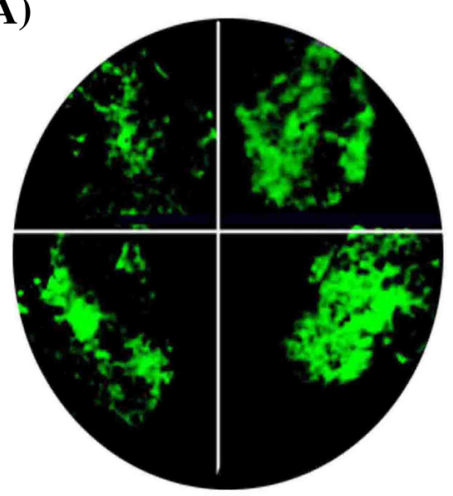

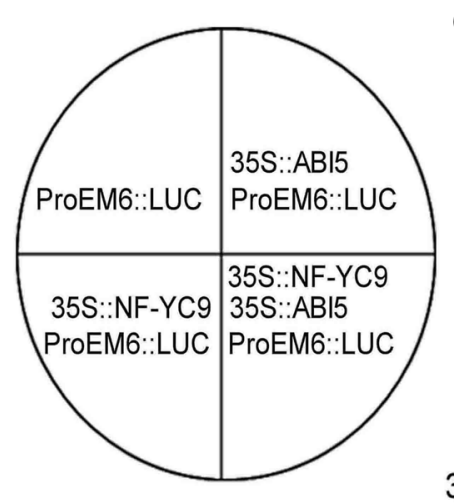

(B)

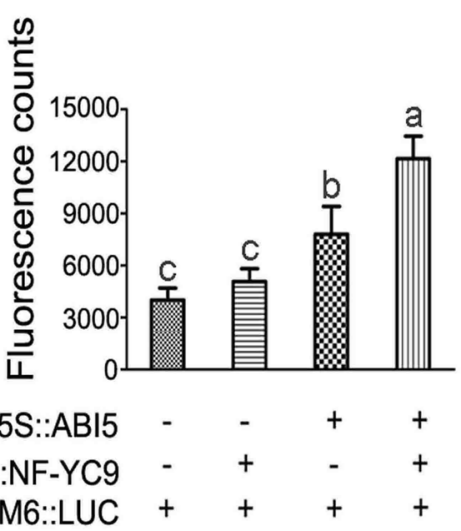

(C)
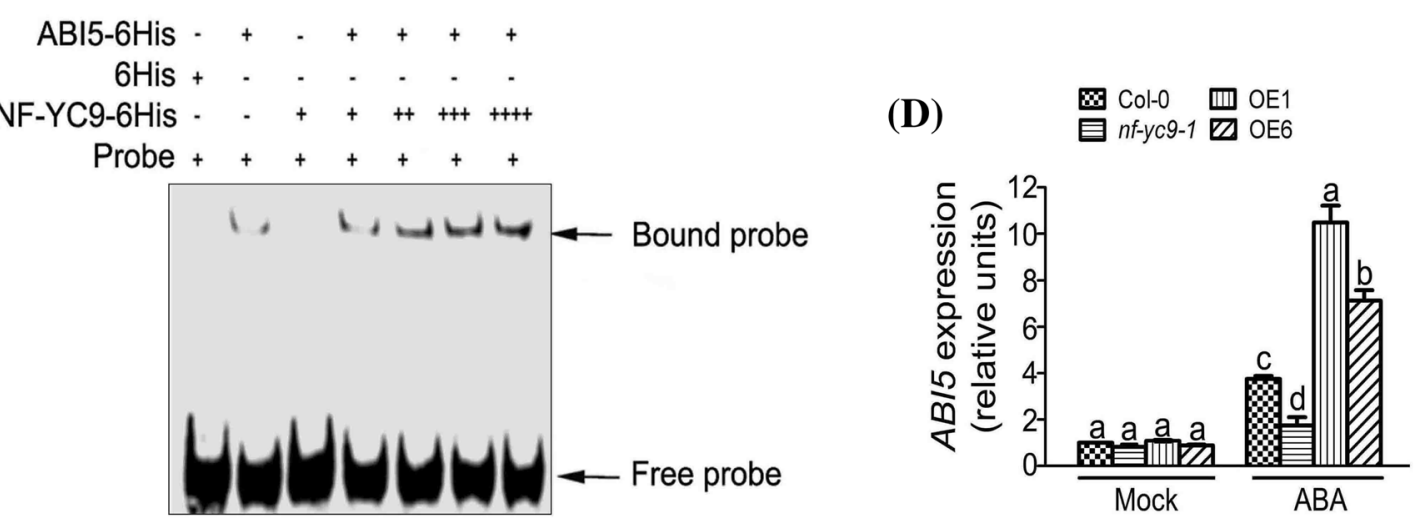

(E)

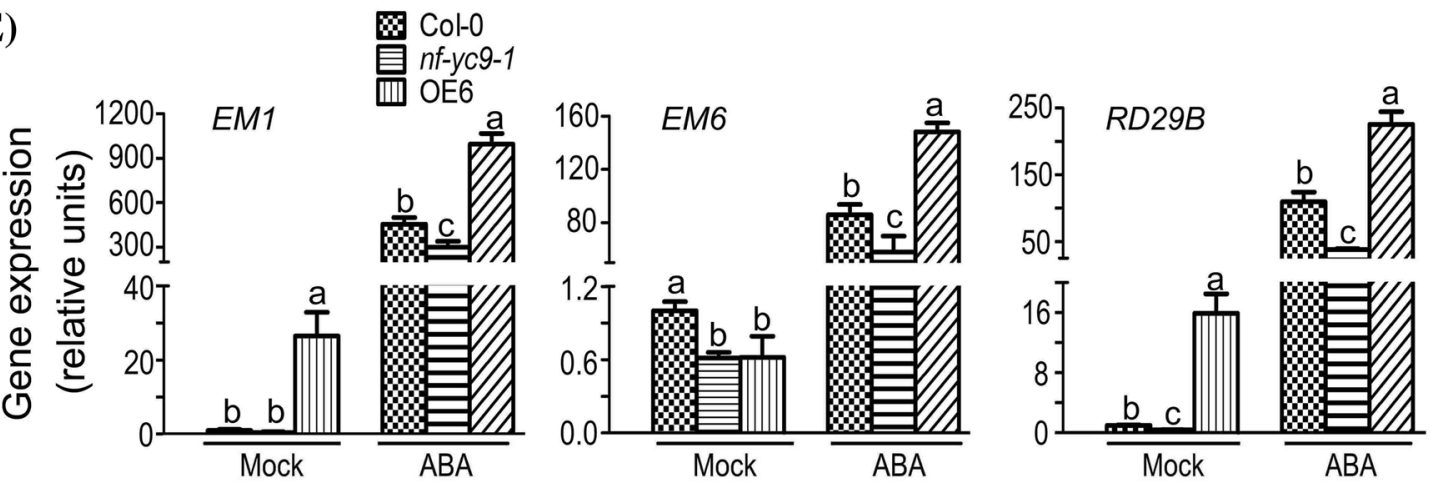

other mutants (Achard et al. 2006). All our data suggest that NF-YC9 may be involved in ABA signaling potentially as a positive regulator, and likely functions redundantly together with other NF-YC members.

\section{How does NF-YC9 work in ABA signaling?}

It has been shown that the plant NF-Y family proteins interact with other transcription factors, which regulate the downstream gene expression, especially those in the bZIP family (Wright et al. 1995; Benatti et al. 2008; Yamamoto et al. 2009; Liu and Howell 2010; Kumimoto et al. 2013). The Arabidopsis NF-YB9/LEC1 and NF-YB6/L1L were shown to interact with bZIP67 to regulate expression of the genes with ABA-responsive elements (ABRE) in their promoters to function in embryogenesis (Yamamoto et al. 2009). In the moss (Physcomitrella patens), NF-YC1 interacts with the ABA-responsive transcription factor ABI3 to form a complex to activate the LEA1, a target gene of ABI3 (Yotsui et al. 2013). In Arabidopsis, three NF-YC members NF-YC3, NF-YC4 and NF-YC9 interact physically with the bZIP transcription factors $\mathrm{ABF} 1$ to $\mathrm{ABF} 4$ and HY5 protein (Kumimoto et al. 2013), which suggests that the NF-YC members may function together with these bZIP transcription factors. However, it has been unknown whether these interaction complexes are associated with regulation of promoter activity or target gene expression. Most recently, Arabidopsis NF-YC3, NF-YC4 and NF-YC9 were shown to interact with 
4Fig. 7 NF-YC9 enhances both activities of ABI5 transcription factor and expression of the $A B I 5$ gene. a Transient expression assays in N. benthamiana leaves, showing that NF-YC9 facilitates ABI5 to activate the target gene EM6 transcription. Representative images of the $N$. Benthamiana leaves $60 \mathrm{~h}$ after infiltration are shown. Tobacco leaves were transformed with the construct combinations as described in the right panel. ProEM6 promoter of the EM6 gene, 35S, 35S promoter; LUC. The experiments were repeated three times with similar results. b Quantitative analyses of the luminescence intensity as shown in (a). The panels show the corresponding quantitative data corresponding to fluorescent images. Each value is the mean $\pm \mathrm{SE}$ with three independent determinations. The symbols "+" and "-" represent that the protein was and was not expressed, respectively, in the tobacco leaves. Each value is the mean \pm SE of three independent biological experiments, and different letters indicate significant differences at $\mathrm{P}<0.05$ (Duncan's multiple range test). c Electrophoretic mobility shift assay, showing that NF-YC9 promotes ABI5 to bind the target gene EM6 promoter. The ABI5 and NF-YC9 proteins were tagged with the $6 \mathrm{His}$ tag peptide (6His). The symbols "+" and "-" represent that the protein was and was not added, respectively, to the EMSA incubation buffer. The NF-YC9-6His protein was added, at a gradient of concentrations, to the EMSA incubation buffer $(1 \mu \mathrm{g}$ for $+; 2 \mu \mathrm{g}$ for $++; 3 \mu \mathrm{g}$ for $+++; 4 \mu \mathrm{g}$ for ++++$)$ to test possible NF-YC9-protein dose-dependence of the ABI5-EM6 promoter interaction. The experiments were repeated three times with the same results. d NF-YC9 stimulates expression of the $A B I 5$ gene: quantitative RT-PCR analysis of the expression levels of $A B I 5$ in the wildtype Col-0, $n f-y c 9-1$ knockdown mutant and the $N F-Y C 9$ overexpression transgenic lines OE1 and OE6 plants in response to ABA treatment. Seedlings of these different genotypes were sprayed with $100 \mu \mathrm{M}( \pm) \mathrm{ABA}$ solution or mock solution $[0 \mu \mathrm{M}( \pm)$ ABA solution, as a control], and sampled for gene expression analysis $4 \mathrm{~h}$ after the ABA treatment. The gene expression levels are normalized relative to the value of wild-type Col-0 plants treated with $0 \mu \mathrm{M}( \pm) \mathrm{ABA}$ treatment, which is taken as 1 . Each value is the mean $\pm \mathrm{SE}$ of three independent biological experiments, and different letters indicate significant differences at $\mathrm{P}<0.05$ (Student's $t$ test) when comparing values within the same treatment. e NF-YC9 stimulates expression of the EM1, EM6 and RD29B genes: quantitative RT-PCR analysis of the expression levels of $E M 1, E M 6$ and $R D 29 B$ in the wild-type Col-0, $n f-y c 9-1$ knockdown mutant and the NF-YC9 overexpression transgenic line OE6 plants in response to ABA treatment. Seedlings of these different genotypes were sprayed with $100 \mu \mathrm{M}( \pm) \mathrm{ABA}$ solution or mock solution [0 $\mu \mathrm{M}( \pm)$ ABA solution, as a control], and sampled for gene expression analysis $4 \mathrm{~h}$ after the ABA treatment. The gene expression levels are normalized relative to the value of wild-type Col-0 plants treated with $0 \mu \mathrm{M}( \pm)$ ABA treatment, which is taken as 1 . Each value is the mean $\pm \mathrm{SE}$ of three independent biological experiments, and different letters indicate significant differences at $\mathrm{P}<0.05$ (Duncan's multiple range test) when comparing values within the same treatment

the DELLA protein RGL2 (a key repressor of GA signaling), forming a NF-YC-RGL2 complex to module ABI5 and thus to regulate ABA signaling in seed germination (Liu et al. 2016). In these events of cell signaling, NF-YC-RGL2 complex functions together as a whole to target the promoter of $A B I 5$ gene, in which RGL2, not itself, but via NF-Y complex, recognizes ABI5 promoter region (Liu et al. 2016). In the present experiment, we showed that NF-YC9 physically interacts with the ABA-responsive bZIP transcription factor ABI5, and facilitates the function of ABI5 to bind and activate the expression of a target gene EM6 (Figs. 6, 7). The expression analysis showed that NF-YC 9 also positively regulates expression of another target gene $E M 1$ of ABI5 as well as an important ABA-responsive gene RD29B (Fig. 7). These data support the model that the NF-YC9, like NF-YB9 and NF-YB6 (Yamamoto et al. 2009) or the moss NF-YC1 (Yotsui et al. 2013), mediates ABA signaling via targeting to and aiding ABA-responsive transcription factors. Our data reveal that NF-YC9 interacts, not only with DELLA protein RGL2 to indirectly regulate ABI5 as previously described (Liu et al. 2016), but also directly with ABI5 itself to modulate function of ABI5, suggesting a different, additional model by which the NF-YC members function to regulate ABI5 in ABA signaling pathway. Noteworthily, we showed that down-regulation of NF-YC9 represses, but overexpression of NF-YC9 enhances, ABA-induced $A B I 5$ expression (Fig. 7d), supporting the idea that NF-YC9 both facilitates ABI5 activity and interacts with other components such as RGL2 (Liu et al. 2016) to directly target to and stimulate $A B I 5$ gene expression in response to ABA. The present findings help to understanding the functional mechanism of the NF-YC proteins in highly complex ABA signaling pathway.

Acknowledgements We thank Dr. Hao Yu (Department of Biological Sciences, National University of Singapore) for the mutant $n f-y c 9-1$ and $N F-Y C 9$ overexpression lines OE1 and OE6. This research was supported by the Grants from the Ministry of Agriculture of China (Grant No. 2016ZX08009003-002) and National Natural Science Foundation of China (Grant No. 31570275).

Author contributions $\mathrm{DPZ}$ and $\mathrm{CB}$ designed experiments and wrote the article. $\mathrm{CB}, \mathrm{YM}$ and $\mathrm{XFW}$ did the experiments.

Open Access This article is distributed under the terms of the Creative Commons Attribution 4.0 International License (http://creativecommons.org/licenses/by/4.0/), which permits unrestricted use, distribution, and reproduction in any medium, provided you give appropriate credit to the original author(s) and the source, provide a link to the Creative Commons license, and indicate if changes were made.

\section{References}

Achard P, Cheng H, De Grauwe L, Decat J, Schoutteten H, Moritz T, Van Der Straeten D, Peng J, Harberd NP (2006) Integration of plant responses to environmentally activated phytohormonal signals. Science 311:91-94

Adie BA, Perez-Perez J, Perez-Perez MM, Godoy M, Sanchez-Serrano JJ, Schmelz EA, Solano R (2007) ABA is an essential signal for plant resistance to pathogens affecting JA biosynthesis and the activation of defenses in Arabidopsis. Plant Cell 19:1665-1681

Benatti P, Basile V, Merico D, Fantoni LI, Tagliafico E, Imbriano C (2008) A balance between NF-Y and p53 governs the proand anti-apoptotic transcriptional response. Nucleic Acids Res 36:1415-1428

Cao S, Kumimoto RW, Gnesutta N, Calogero AM, Mantovani R, Holt BF (2014) A distal CCAAT/NUCLEAR FACTOR Y complex 
promotes chromatin looping at the FLOWERING LOCUS promoter and regulates the timing of flowering in Arabidopsis. Plant Cell 26:1009-1017

Carles C, Bies-Etheve N, Aspart L, Léon-Kloosterziel KM, Koornneef M, Echeverria M, Delseny M (2002) Regulation of Arabidopsis thaliana Em genes: role of ABI5. Plant J 30:373-383

Chen H, Zou Y, Shang Y, Lin H, Wang Y, Cai R, Tang X, Zhou JM (2008) Firefly luciferase complementation imaging assay for protein-protein interactions in plants. Plant Physiol 146:368-376

Chen Q, Sun J, Zhai Q, Zhou W, Qi L, Xu L, Wang B, Chen R, Jiang H, Qi J, Li X, Palme K, Li C (2011) The basic helix-loop-helix transcription factor MYC2 directly represses PLETHORA expression during jasmonate mediated modulation of the root stem cell niche in Arabidopsis. Plant Cell 23:3335-3352

Cutler SR, Rodriguez PL, Finkelstein RR, Abrams SR (2010) Abscisic acid: emergence of a core signaling network. Annu Rev Plant Biol 61:651-679

Finkelstein RR, Lynch TJ (2000) The Arabidopsis abscisic acid response gene $A B I 5$ encodes a basic leucine zipper transcription factor. Plant Cell 12:599-609

Finkelstein RR, Gampala SS, Rock CD (2002) Abscisic acid signaling in seeds and seedlings. Plant Cell 14:S15-S45

Gusmaroli G, Tonelli C, Mantovani R (2001) Regulation of the CCAAT-binding NF-Y subunits in Arabidopsis thaliana. Gene 264:173-185

Hou X, Zhou J, Liu C, Liu L, Shen L, Yu H (2014) Nuclear factor Y-mediated H3K27me3 demethylation of the SOC1 locus orchestrates flowering responses of Arabidopsis. Nat Commun 5:4601. doi: $10.1038 /$ ncomms 5601

Jiang SC, Mei C, Liang S, Yu YT, Lu K, Wu Z, Wang XF, Zhang DP (2015) Crucial roles of the pentatricopeptide repeat protein SOAR1 in Arabidopsis response to drought, salt and cold stresses. Plant Mol Biol 88:369-385

Kumimoto RW, Siriwardana CL, Gayler KK, Risinger JR, Siefers N, Holt BF (2013) NUCLEAR FACTOR Y transcription factors have both opposing and additive roles in ABA-mediated seed germination. PLoS ONE 8:e59481

Kwak JM, Ma"ser P, Schroeder JH (2008) The clickable guard cell,version II: interactive model of guard cell signal transduction mechanisms and pathways. Arabidopsis Book Am Soc Plant Biol. doi:10.1199/tab.0099

Laloum T, De Mita S, Gamas P, Baudin M, Niebel A (2013) CCAATbox binding transcription factors in plants: $\mathrm{Y}$ so many? Trends Plant Sci 18:157-166

Leyva-González MA, Ibarra-Laclette E, Cruz-Ramírez A, HerreraEstrella L (2012) Functional and transcriptome analysis reveals an acclimatization strategy for abiotic stress tolerance mediated by Arabidopsis NF-YA family members. PLoS ONE 7:e48138

Li WX, Oono Y, Zhu J, He XJ, Wu JM, Iida K, Lu XY, Cui X, Jin H, Zhu JK (2008) The Arabidopsis NF-YA5 transcription factor is regulated transcriptionally and posttranscriptionally to promote drought resistance. Plant Cell 20:2238-2251

Li YJ, Fang Y, Fu YR, Huang JG, Wu CA, Zheng CC (2013) NF-YAl is involved in regulation of postgermination growth arrest under salt stress in Arabidopsis. PLoS ONE 8:e61289

Liu JX, Howell SH (2010) bZIP28 and NF-Y transcription factors are activated by ER stress and assemble into a transcriptional complex to regulate stress response genes in Arabidopsis. Plant Cell 22:782-796

Liu ZQ, Yan L, Wu Z, Mei C, Lu K, Yu YT, Liang S, Zhang XF, Wang XF, Zhang DP (2012) Cooperation of three WRKY-domain transcription factors WRKY18, WRKY40, and WRKY60 in repressing two ABA-responsive genes ABI4 and ABI5 in Arabidopsis. J Exp Bot 63:6371-6392

Liu R, Xu YH, Jiang SC, Lu K, Lu YF, Feng XJ, Wu Z, Liang S, Yu YT, Wang XF, Zhang DP (2013) Light-harvesting chlorophyll a/b binding proteins, positively involved in abscisic acid signalling, require a transcription repressor, WRKY40, to balance their function. J Exp Bot 64:5443-5456

Liu X, Hu P, Huang M, Tang Y, Li Y, Li L, Hou X (2016) The NF-YCRGL2 module integrates GA and ABA signalling to reg ulate seed germination in Arabidopsis. Nat Commun 7:12768. doi:10.1038/ ncomms 12768

Mantovani R (1999) The molecular biology of the CCAAT-binding factor NF-Y. Gene 239:15-27

Mu J, Tan H, Hong S, Liang Y, Zuo J (2013) Arabidopsis transcription factor genes $N F-Y A 1,5,6$, and 9 play redundant roles in male gametogenesis, embryogenesis, and seed development. Mol Plant 6:188-201

Nelson DE, Repetti PP, Adams TR, Creelman RA, Wu J, Warner DC, Anstrom DC, Bensen RJ, Castiglioni PP, Donnarummo MG, Hinchey BS, Kumimoto RW, Maszle DR, Canales RD, Krolikowski KA, Dotson SB, Gutterson N, Ratcliffe OJ, Heard JE (2007) Plant nuclear factor Y (NF-Y) B subunits confer drought tolerance and lead to improved corn yields on water-limited acres. Proc Natl Acad Sci USA 104:16450-16455

Park SY, Fung P, Nishimura N, Jensen DR, Fujii H, Zhao Y, Lumba S, Santiago J, Rodrigues A, Chow TF, Alfred SE, Bonetta D, Finkelstein R, Provart NJ, Desveaux D, Rodriguez PL, McCourt P, Zhu JK, Schroeder JI, Volkman BF, Cutler SR (2009) Abscisic acid inhibits type $2 \mathrm{C}$ protein phosphatases via the PYR/PYL family of START proteins. Science 324:1068-1071

Petroni K, Kumimoto RW, Gnesutta N, Calvenzani V, Fornari M, Tonelli C, Holt BF, Mantovani R (2012) The promiscuous life of plant NUCLEAR FACTOR Y transcription factors. Plant Cell 24:4777-4792

Sato H, Mizoi J, Tanaka H, Maruyama K, Qin F, Osakabe Y, Morimoto K, Ohori T, Kusakabe K, Nagata M, Shinozaki K, YamaguchiShinozaki K (2014) Arabidopsis DPB3-1, a DREB2A interactor, specifically enhances heat stress-induced gene expression by forming a heat stress-specific transcriptional complex with NF-Y subunits. Plant Cell 26:4954-4973

Shang Y, Yan L, Liu ZQ, Cao Z, Mei C, Xin Q, Wu FQ, Wang XF, Du SY, Jiang T, Zhang XF, Zhao R, Sun HL, Liu R, Yu YT, Zhang DP (2010) The Mg-chelatase $\mathrm{H}$ subunit of Arabidopsis antagonizes a group of WRKY transcription repressors to relieve ABA-responsive genes of inhibition. Plant Cell 22:1909-1935

Shi H, Ye T, Zhong B, Liu X, Jin R, Chan Z (2014) AtHAP5A modulates freezing stress resistance in Arabidopsis through binding to CCAAT motif of AtXTH21. New Phytol 203:554-567

Shinozaki K, Yamaguchi-Shinozaki K (2007) Gene networks involved in drought stress response and tolerance. J Exp Bot 58:221-227

Shinozaki K, Yamaguchi-Shinozaki K, Seki M (2003) Regulatory network of gene expression in the drought and cold stress responses. Curr Opin Plant Biol 6:410-417

Siefers N, Dang KK, Kumimoto RW, Bynum WE, Tayrose G, Holt BF (2009) Tissue-specific expression patterns of Arabidopsis NF-Y transcription factors suggest potential for extensive combinatorial complexity. Plant Physiol 149:625-641

Siriwardana CL, Kumimoto RW, Jones DS, Holt BF (2014) Gene family analysis of the Arabidopsis NF-YA transcription factors reveals opposing abscisic acid responses during seed germination. Plant Mol Biol Rep 32:971-986

Stephenson TJ, McIntyre CL, Collet C, Xue GP (2007) Genome-wide identification and expression analysis of the NF-Y family of transcription factors in Triticum aestivum. Plant Mol Biol 65:77-92

Tang Y, Liu X, Liu X, Li Y, Wu K, Hou X (2017) Arabidopsis NF-YCs mediate the light-controlled hypocotyl elongation via modulating histone acetylation. Mol Plant 10:260-273

Warpeha KM, Upadhyay S, Yeh J (2007) The GCR1, GPA1, PRN1, NF-Y signal chain mediates both blue light and abscisic acid responses in Arabidopsis. Plant Physiol 143:1590-1600 
Wright KL, Moore TL, Vilen BJ, Brown AM, Ting JPY (1995) Major histocompatibility complex class II-associated invariant chain gene expression is up-regulated by cooperative interactions of Sp1 and NF-Y. J Biol Chem 270:20978-20986

Wu FQ, Xin Q, Cao Z, Liu ZQ, Du SY, Mei C, Zhao CX, Wang XF, Shang Y, Jiang T, Zhang XF, Yan L, Zhao R, Cui ZN, Liu R, Sun HL, Yang XL, Su Z, Zhang DP (2009) The magnesium-chelatase $\mathrm{H}$ subunit binds abscisic acid and functions in abscisic acid signaling: new evidence in Arabidopsis. Plant Physiol 150:1940-1954

Yamamoto A, Kagaya Y, Toyoshima R, Kagaya M, Takeda S, Hattori T (2009) Arabidopsis NF-YB subunits LEC1 and LEC1-LIKE activate transcription by interacting with seed-specific ABREbinding factors. Plant J 58:843-856

Yeap WC, Lee FC, Shabari Shan DK, Musa H, Appleton DR, Kulaveerasingam H (2017) WRI1-1, ABI5, NF-YA3 and NF-YC2 increase oil biosynthesis in coordination with hormonal signaling during fruit development in oil palm. Plant J 91(1):97-113. doi:10.1111/tpj.13549

Yotsui I, Saruhashi M, Kawato T, Taji T, Hayashi T, Quatrano RS, Sakata Y (2013) ABSCISIC ACID INSENSITIVE3 regulates abscisic acid-responsive gene expression with the nuclear factor Y complex through the ACTT-core element in Physcomitrella patens. New Phytol 199:101-109

Zhai Q, Yan L, Tan D, Chen R, Sun J, Gao L, Dong MQ, Wang Y, Li C (2013) Phosphorylation-coupled proteolysis of the transcription factor MYC2 is important for jasmonate-signaled plant immunity. PLoS Genet 9:e1003422

Zhu JK (2002) Salt and drought stress signal transduction in plants. Annu Rev Plant Biol 53:247-273

Zhu JK (2003) Regulation of ion homeostasis under salt stress. Curr Opin Plant Biol 6:441-445 